\title{
Serum Zinc Deficiency in Squamous Cell Carcinomas of Oral and Maxillofacial Region: A Risk Factor to its Development and Metastasis
}

Zhenq Xu ( $\sim$ hugoxuarticles@hotmail.com )

Tianjin Stomatological Hospital https://orcid.org/0000-0001-5254-6724

Jianw Shang

Tianjin Stomatological Hospital

Guoq Wang

Tianjin Stomatological Hospital

Li Long

Tianjin Stomatological Hospital

Ping Zhang

Tianjin Stomatological Hospital

Research article

Keywords: serum Zinc deficiency, squamous cell carcinoma, predisposing factor, progression, metastasis

Posted Date: May 14th, 2020

DOl: https://doi.org/10.21203/rs.3.rs-27684/v1

License: (a) (i) This work is licensed under a Creative Commons Attribution 4.0 International License.

Read Full License 


\section{Abstract}

Objectives To explore whether serum Zinc deficiency (sZnd) is a promoter to the development and lymphonode metastasis of squamous cell carcinoma (SCC) of oral and maxillofacial region (OMF).

Methods 610 patients with SCC were enrolled in the study group, the controls consisted of the same number of age-gender matched subjects without cancers, serum Zinc level was tested in both groups. Characteristics of SCCs were investigated. Different concentration of serum Zinc and prevalence of sZnd between the two groups were calculated using Chi-square test. The association between serum Zinc status and SCC with different T-stages and lymphonode metastasis was focused on research.

Results The patients varied from 19 to 87 years with a mean age of $58.89 \pm 10.91$ years. Oral cavity was the most commonly affected region. Males were involved predominantly with a ratio of $1.44: 1$ to females. The concentration of serum Zinc in malignancies was $12.57 \pm 2.53 \mu \mathrm{mol} / \mathrm{L}, 19.2 \%$ suffered from sZnd; in the control group were 14.65 $\pm 2.25 \mu \mathrm{mol} / \mathrm{L}$ and 6.1\%. Decreased serum Zinc and increased prevalence of sZnd in SCC were statistically significant to controls $(p=0.000)$. Increased incidence of sZnd and reduced level of serum Zinc correlated significantly to the the progression and lymphnode metastasis of SCC $(p<0.01)$.

Conclusions sZnd was a predisposing indicator to the tumorigenesis of SCC and also a promoter to its aggravation and lymphonode metastasis, which should be considered as a factor in devising the treatment plannings.

\section{Introduction}

Oral and maxillofacial region (OMF) encompasses majority of head and neck, including the lip, oral cavity, maxillary sinus, oropharynx, and salivary glands. There are several types of cancers involved OMF, such as teratoma, adenocarcinoma derived from salivary gland, lymphoma, or melanoma, but around $90 \%$ are squamous cell carcinomas (SCC) which often spread to the lymph nodes of the neck [1].

The exact pathogenesis of SCC has not been clearly elucidated till date. However, it is strongly associated with certain environmental or lifestyle risk factors, including tobacco smoking, alcohol consumption, particular chemicals used in certain workplaces, and certain strains of viruses, such as human papillomavirus [2].

Trace elements (including Zinc, Selenium and Cuprum) play an important role in maintaining the health of human body, dysregulation of them may result in disease and impaired metabolism. Trace element abnormalities, especially Zinc deficiency (Znd), have been reported in many types of cancers and implicated in their oncogenesis (such as prostate cancer and esophageal cancer) in few pilot articles [3]. However, it has received relatively little research attention to oral and maxillofacial region. 
A retrospective study conducted in our research aims to investigate the relationship between serum Zinc deficiency (sZnd) and the development of SCC of OMF. The association between the reduction of serum Zinc status and different T-stages and the risk of lymph node metastasis of SCC was focused on research.

\section{Materials And Methods}

\section{Serum Zinc measurement}

Blood biochemical analysis (including trace element detection) of patients were taken as a routine examination when they were admitted to the department of oral and maxillofacial surgery of Tianjin Stomatological Hospital of Nankai University. The normal level of serum Zinc ranges from 10.8 to $19.7 \mu \mathrm{mol} / \mathrm{L}$. Anyone whose serum Zinc level belowed $10.8 \mu \mathrm{mol} / \mathrm{L}$ was detected as having sZnd.

\section{Research groups}

The study involved a SCC group and a control group, both of which were selected from inpatient electronic medical record database of our hospital from August 2016 to February 2020. The research was approved by the Tianjin Stomatological Hospital Ethics Committee.

610 patients pathologically diagnosed as SCC by the faculty were selected. Characteristics of age, gender, affected region, concentration of serum Zinc and prevalence of sZnd were analyzed. For the control group, the same number of persons was chosen randomly by searching the remaining non-tumor electronic medical records based on age and gender matching. The concentration of serum Zinc and prevalence of sZnd were also accounted. Patients suffering from eating difficulty $\square$ dysphagia $\square$ malnutrition or cachexia were excluded in both groups.

The alteration of serum Zinc level between the 2 groups was analyzed. The difference in prevalence of sZnd in malignant cases compared with controls and the odds ratio of having sZnd in SCC were calculated.

\section{Subset groups of SCC}

The objectives with SCC were divided into 4 groups according to WHO T-stage standard. In the same way, patients were divided into 2 groups based on cervical lymph node metastasis or not [4]. The difference in the prevalence of sZnd and concentration of serum Zinc among different groups were analyzed.

\section{Statistical analysis}

The analyses were performed using SPSS 22.0 software. Differences among the T-staging groups were assessed using the Tukey range test

post hoc t-tests for multiple comparisons. When there were only two groups, a standard t-test was used to analyze the data. Chi-square test was performed to calculate the $P$ value. An $\otimes$ level of 0.05 was 


\section{Results}

\section{Characteristics of SCC of OMF}

The age of patients with SCC varied from 19 to 87 years (mean age was $58.89 \pm 10.91$ years) with a peak age of 45-74 years. Oral cavity was the most commonly affected primary region, followed by lip and oral pharynx. Males were involved predominantly with a ratio of 1.44:1 to females (Shown in Table-1).

Table 1

Characteristics of SCC of OMF

\begin{tabular}{|lll|}
\hline Site,Sex Age(y) & $\begin{array}{l}\text { LP OC MS OP SG } \\
\text { M F M F M F M F M F }\end{array}$ & Sum \\
\hline$\leqq 25$ & 3 & 3 \\
\hline $26-44$ & 3292721 & 62 \\
\hline $45-59$ & 15910776172 & 217 \\
\hline $60-74$ & 27181181002372 & 295 \\
\hline $75-89$ & 521871 & 33 \\
\hline sum & 5029272 213 0133750 & 610 \\
\hline $\begin{array}{l}\text { ( Abbreviations: SCC-squamous cell carcinoma; OMF-oral and maxillofacial region; LP-lip; OC-oral } \\
\text { cavity; MS-maxillary sinus; OP-oral pharynx; SG-salivary gland; M-male; F-female. ) }\end{array}$ \\
\hline
\end{tabular}

\section{Serum Zinc status in malignant and control groups}

The concentration of serum Zinc in SCC group is $12.57 \pm 2.53 \mu \mathrm{mol} / \mathrm{L}$, much decreased than controls of $14.65 \pm 2.25 \mu \mathrm{mol} / \mathrm{L}$, and the difference was statistically significant $(P=0.000) .117$ patients suffered from sZnd in SCC, and thus the prevalence of sZnd in research group was 19.2\%; while, in the control group, 37 patients were affected by sZnd, therefore, the prevalence of sZnd in controls was $6.1 \%$. The difference in the prevalence/incidence of sZnd in malignant cases versus controls was found to be statistically significant using Chi-square test $(P=0.000)$. Relative risk of developing SCC of OMF in persons with sZnd is 3.68 (95\% confidence interval $2.49-5.42$ ) compared to those with normal serum Zinc (Shown in Table-2). 
Table 2

Prevalence of sZnd and concentration of serum Zinc level in SCC and control groups

\begin{tabular}{|c|c|}
\hline Group & sCC Control \\
\hline Prevalence of sZnd (case) & $117 \rrbracket 61037 \rrbracket 610 *$ \\
\hline Concentration of serum Zinc $(\mu \mathrm{mol} / \mathrm{L})$ & $14.65 \pm 2.2512 .57 \pm 2.53^{*}$ \\
\hline \multicolumn{2}{|c|}{ (Abbreviations: sZnd-serum Zinc deficiency; SCC-squamous cell carcinoma; ${ }^{*} \mathbb{p} \otimes 0.01, \# \mathrm{p} \otimes 0.05$ ) } \\
\hline
\end{tabular}

According to WHO T-stage standard, patients with SCC were divided into 4 groups: T1 $1 \mathrm{TT} 2 \mathrm{TT} 3$ and T4.

Concentration of serum Zinc decreased with the development of T-stage, and the difference was statistically significant between early stage ( T1 TT2) and late stage ( T3ロT4 ), while no significant difference was observed within T1 vs T2 and T3 vs T4 groups $(p=0.000$, Shown in Table-3).

Prevalence of sZnd significantly increased in late stage ( T3ロT4 ) than early stage ( T10T2 ) using Chisquare test $(p=0.000$, Shown in Table-3).

Table 3

Prevalence of sZnd and concentration of serum Zinc level in T1-T4 stage of SCC

\begin{tabular}{|c|c|c|}
\hline \multirow[t]{2}{*}{ Group } & Early stage & Late stage \\
\hline & T1 T2 & T3*@ T4*@ \\
\hline Prevalence of sZnd (case) & $26 \rrbracket 17435 \llbracket 234$ & $26 \rrbracket 10130 \rrbracket 101$ \\
\hline Concentration of serum Zinc ( $\mu \mathrm{mol} / \mathrm{L})$ & $13.10 \pm 2.3412 .77 \pm 2.55$ & $12.14 \pm 2.11^{\star \&} 11.63 \pm 2.86^{\star} @$ \\
\hline
\end{tabular}

\section{Serum Zinc status in lymph node metastasis of SCC}

2 groups were gained on the basis of lymph node metastasis (N1) or not (N0) in patients with SCC.

The concentration of serum Zinc in group with lymph node metastasis is $11.70 \pm 2.72 \mu \mathrm{mol} / \mathrm{L}$, while $12.77 \pm 2.44 \mu \mathrm{mol} / \mathrm{L}$ in group without metastasis. Reduction in serum Zinc level correlated with the metastasis of lymph node metastasis, and the difference was statistically significant $(p=0.001$, Shown in Table-4). 
Table 4

Prevalence of sZnd and concentration of serum Zinc level in SCC with lymph node metastasis or free

Group

Prevalence of sZnd (case)

Concentration of serum Zinc ( $\mu \mathrm{mol} / \mathrm{L})$
N0 N1

$83 \rrbracket 49834 \rrbracket 112^{\star}$

$12.77 \pm 2.4411 .7 \pm 2.72 *$

( Abbreviations: sZnd-serum Zinc deficiency; SCC-squamous cell carcinoma; N0-non lymph node metastasis group; N1-lymph node metastasis group; * p囚0.01 )

Significant increased prevalence of sZnd correlated to cervical lymph node metastasis using Chi-square test ( $p=0.001$, Shown in Table-4). Relative risk of having metastasis in SCC patients suffering from sZnd is 2.18 (95\% confidence interval 1.37-3.48) versus those with normal serum Zinc.

\section{Discussion}

Oral and maxillofacial malignancy is one of the most lethal tumors and a major leading cause of mortality due to local recurrence or metastasis, however, there is lack of epidemiological data on its exact prevalence. An estimated 300,400 new cases and 145,400 deaths from oral cavity cancer (including lip cancer) occurred in 2012 worldwide [5], and in China an estimated annual incidence of 1.6-1.9 per 100,000 persons was assessed [6]. The usual age at diagnosis of oral cancer is between 55 and 65 years old and males are affected twice as often as females and SCC is most likely to appear in males over 40 years of age with a history of heavy alcohol use coupled with smoking [7].

In our study about SCC in OMF, males are more involved than females with a ratio of 1.44:1, with a peak age of $45-74$ years. Oral cavity is the most commonly affected primary region accounting for $79.51 \%$ of all the cases, followed by lip and oral pharynx.

Zinc is an essential trace element which can act as a co-factor for more than 300 enzymes that regulate a variety of cellular processes and signaling pathways [8]. The imbalance of Zinc homeostasis has been established in varied pathological conditions, including many types of cancer, such as prostate cancer, breast cancer, lung cancer and ovarian cancer [3].

There are three main parameters commonly used to detect Zinc level of human body: tissue Zinc, serum Zinc and excrement Zinc. Tissue Zinc is more specific and sensitive to indicate the alternation of Zinc level, however, it needs a high quality of experimental condition and is relatively complicated to operate. The utility of excrement Zinc as a measure of Zinc status, such as urinary Zinc, is also limited by the difficulties encountered in collecting a $24 \mathrm{~h}$ specimen without exogenous Zinc contamination [9]. Blood is easy to be harvested and many studies provide evidence that the trend of changes of Zinc level in tissue and serum is consistent, which makes serum Zinc to be an acceptable clinical index to demonstrate the alteration of Zinc status [3]. In our study, serum Zinc was applied to indicate the Zinc status of human body. 
Despite current advances in its fundamental research and clinical management, the OMF cancer remains a difficult region to control. Surgery is still the most important treatment method in SCC of OMF, cancer can be highly curable if detected early, while it is hard to excise completely due to the restriction of anatomy and tissue defects created by extensive resection can severely affect speech and swallowing function as well as destroy the aesthetic appearance at late stage. A 5-year survival remains poor at $62.7 \%$ in the USA and below $50 \%$ in other countries due to local recurrence and distant metastatic spread even the combination of chemotherapy and radiotherapy were applied in patients $[10,11]$. Thus, further study into its pathogenesis and predictors of outcome is critical to devise a new preventive measure, clarify an earlier diagnosis, and offer a novel treatment modality to SCC of OMF.

In our study, the concentration of serum Zinc in SCC decreased significantly compared to the controls. Increased prevalence of sZnd in SCC group was observed and calculated to be statistically significant versus controls. Serum Zinc level reduced and incidence of sZnd increased were observed with the progression of tumor grade, the difference were statistically significant between early stage ( T10T2) and late stage ( T3ロT4 ) using Chi-square test. SCC of OMF has a propensity for cervical nodal spread. In our research we found that concentration of serum Zinc in SCCs accompanied lymph node metastasis decreased significantly to those without metastasis. SCC patients suffering from sZnd had a more higher risk of having metastasis compared with those with normal serum Zinc. The results above demonstrated that decreased serum Zinc level, especially sZnd, was a predisposing indicator to the tumorigenesis of SCC, and also a promoter to its aggravation and lymph node metastasis.

sZnd has been proved significantly associated to the development and aggravation of SCC of OMF in our study, however, the exact mechanism remains poorly uncovered. Up to date, the underlying mechanisms may include: $\nabla$ Zinc serves as a co-factor in Zinc-dependent matrix metalloproteinases which augment autodebridement and keratinocyte migration during wound repair and its importance in wound healing is well appreciated $[12,13]$. The most common clinical presentation of SCC of OMF is an incurable ulcer, such as tongue cancer, buccal cancer and mouth floor cancer [14], Zinc deficiency in tumor microenvironment may delay wound healing and lead to pathological changes of SCC. Zinc deficiency is commonly detected in patients with recurrent aphthous ulceration (RAU), and improvement in oral wound healing rates and reduction in the recurrence rate have been observed clinically in RAU via systematic Zinc supplementation $[15,16]$, thus Zinc administration could be a novel anticancer therapeutic candidate in SCC; $\otimes$ Multiple lifestyle or environmental factors, such as tobacco use, alcohol abuse, chemical exposure and virus infection, can cause DNA damage and abnormalities of genome integrity. Zinc plays an important role in transcription factor function, antioxidant defense and DNA repair. Deficiencies in Zinc can contribute to single- and double-strand DNA breaks and oxidative modifications to DNA that increase risk for cancer development [17]. Oncogenesis of SCC occurs as a result of the accumulation of gene and chromosome mutations [18]. Benefits maybe gained from Zinc supplement through repairing DNA damage and maintaining integrity of chromosomes and genes in patients with SCC; $\triangle$ Zinc deficiency has been well documented in many cancers with elevated level of Hedgehog $(\mathrm{Hh})$ ligand both in patient serum and affected tissues. When Zinc levels are low, Hh autoprocessing may be enhanced, leading to overproduction of $\mathrm{Hh}$ ligand and potentially abnormal activation of Hh signaling 
pathway [19]. Hedgehog $(\mathrm{Hh})$ signaling pathway stimulation has been observed in oral squamous cell carcinomas and this low Zinc-high Hh axis may contribute to its pathogenesis [20]. Thus cancer prevention could be achieved by Zinc fuels that Zinc can bind to the active site of the Hedgehog-intein (Hint) domain and inhibit $\mathrm{Hh}$ ligand production; $\mathbb{Q} \mathrm{It}$ is well established that there exist comprehensive interactions between tumor immunology and tumor biology in head and neck squamous cell carcinoma [21]. Zinc is required for the activity of many enzymes for proper immune function. Zinc deficiency affects multiple aspects of innate and adaptive immunity, including thymic atrophy, altered thymic hormones, lymphopenia, and compromised cellular-and antibody-mediated responses that reduces the lytic activity of natural killer cells, impairs NKT cell cytotoxicity and immune signaling [22]. Zinc supplementation might either restore function in the setting of dysfunction or improve normal immune cell function in SCC which would be contributed to the development of customized treatment strategy.

\section{Conclusion}

The relationship between decreased serum Zinc level and development of SCC has been revealed in our study, and sZnd has been proved significantly associated to its aggravation and metastasis. Thus, reduction in serum Zinc status should be considered as an indicator to predict its aggressive progression and poor prognosis in patients with SCC, and it also should be emphasized in making the treatment decisions, especially to those suffering from sZnd. From therapeutic aspect, with a better understanding of how sZnd induces SCC in the future, Zinc supplementation may have a role in the prevention and maybe become an adjuvant therapy to its treatment of SCC.

\section{Declarations}

Ethics approval and consent to participate: The study was approved by the Tianjin Stomatological Hospital Ethics Committee,and informed consent written was obtained from all participants.

Consent for Publication: Not applicable

Availability of data and material: We declared that materials described in the manuscript, including all relevant raw data, will be freely available to any scientist wishing to use them for non-commercial purposes, without breaching participant confidentiality.

Competing interests: The authors declare that they have no competing interests.

Funding: Not applicable

Authors' contributions:

ZX, designs the study and writes the paper;

JS, confirm the histology type of oral cancer;

GW, doses the test of serum Zinc;

LL and PZ, collect data . 
Acknowledgements: Much thanks to Dr. Xu Xiang, Hui Jiang, Yingb Yan and Jun Zhang for the supplies of medical records.

\section{References}

1. Argiris A, Karamouzis MV, Raben D, et al. Head and neck cancer. Lancet. 2008;371(9625):1695-709.

2. Li S, Lee YC, Li Q, et al. Oral lesions, chronic diseases and the risk of head and neck cancer. Oral Oncol. 2015;51(12):1082-7.

3. Gumulec J, Masarik M, Adam V, et al. Serum and tissue zinc in epithelial malignancies: a metaanalysis. PLoS One. 2014;9(6):e99790.

4. Huang SH, O'Sullivan B. Overview of the 8th Edition TNM Classification for Head and Neck Cancer. Curr Treat Options Oncol. 2017;18(7):40.

5. Torre LA, Bray F, Siegel RL, et al.

6. Global cancer statistics. 2012. CA Cancer J Clin. 2015;65(2):87-108.

7. Zhang Zhiy YG. Oral and Maxillofacial Surgery. 7th ed. Bei Jing: People's Medical Publishing House; 2015. p. 281.

8. Petersen PE. Oral cancer prevention and control-the approach of the World Health Organization. Oral Oncol. 2009;45(4-5):454-60.

9. Maret W, Li Y. Coordination Dynamics of Zinc in Proteins. Chem Rev. 2009;109(10):4682-707.

10. Christudoss P, Selvakumar R, Fleming JJ, et al. Zinc status of patients with benign prostatic hyperplasia and prostate carcinoma. Indian J Urol. 2011;27(1):14-8.

11. Prince S, Bailey BM. Squamous carcinoma of the tongue: review. Br J Oral Maxillofac Surg. 1999;37(3):164-74.

12. Kolokythas A, Park S, Schlieve T, et al. Squamous cell carcinoma of the oral tongue: histopathological parameters associated with outcome. Int J Oral Maxillofac Surg. 2015;44(9):1069-74.

13. Lansdown AB. Zinc in the healing wound. Lancet. 1996;347(9003):706-7.

14. Kogan S, Sood A, Garnick MS. Zinc and Wound Healing: a review of Zinc physiology and clinical applications. Wounds. 2017;29(4):102-6.

15. Markopoulos AK. Current aspects on oral squamous cell carcinoma. Open Dent J. 2012;6:126-30.

16. Orbak R, Cicek $Y$, Tezel A, et al. Effects of zinc treatment in patients with recurrent aphthous stomatitis. Dent Mater J. 2003;22(1):21-9.

17. Ozler GS. Zinc deficiency in patients with recurrent aphthous stomatitis: a pilot study. J Laryngol Otol. 2014;128(6):531-3.

18. Ho E. Zinc deficiency, DNA damage and cancer risk. J Nutr Biochem. 2004;15(10):572-8.

19. Scully C, Field JK, Tanzawa H. Genetic aberrations in oral or head and neck squamous cell carcinoma (SCCHN): 1. Carcinogen metabolism, DNA repair and cell cycle control. Oral Oncol. 
2000;36(3):256-63.

20. Xie J, Owen T, Xia K, et al. Zinc inhibits Hedgehog autoprocessing: linking zinc deficiency with Hedgehog activation. J Biol Chem. 2015;290(18):11591-600.

21. Kuroda H, Kurio N, Shimo T, et al. Oral Squamous Cell Carcinoma-derived Sonic Hedgehog Promotes Angiogenesis. Anticancer Res. 2017;37(12):6731-7.

22. Jin Y, Qin X. Profiles of immune cell infiltration and their clinical significance in head and neck squamous cell carcinoma. Int Immunopharmacol. 2020;82:106364.

23. John E, Laskow TC, Buchser WJ, et al. Zinc in innate and adaptive tumor immunity. J Transl Med. 2010;8:118. 